\title{
Case Report Diagnosing of Imperforate Hymen by Ultrasound
}

\author{
Ala Mohammed Abd Elgyoum ${ }^{1,2, *}$, Elrashed Abd Elrahim Mohammed ${ }^{1,3}$, \\ Ahmed Abdelrahim Mohammed ${ }^{3}$ \\ ${ }^{1}$ College of Applied Medical Science, Taif University, Taif, Saudi Arabia \\ ${ }^{2}$ Faculty of Radiological and Nuclear Medicine Science, National Ribat University, Khartoum, Sudan \\ ${ }^{3}$ Faculty of Radiology Science and Medical Imaging, Alzaiem Alazhari University, Khartoum North, Sudan
}

Email address:

alas-sa@hotmail.com (A. M. A. Elgyoum)

${ }^{*}$ Corresponding author

\section{To cite this article:}

Ala Mohammed Abd Elgyoum, Elrashed Abd Elrahim Mohammed, Ahmed Abdelrahim Mohammed. Case Report Diagnosing of Imperforate Hymen by Ultrasound. International Journal of Biomedical Materials Research. Vol. 4, No. 3, 2016, pp. 27-30. doi: 10.11648/j.ijbmr.20160403.13

Received: September 26, 2016; Accepted: November 4, 2016; Published: November 29, 2016

\begin{abstract}
An imperforate hymen is a congenital disorder where a hymen without an opening completely obstructs the vagina. A16 year old, female, single with cyclic, lower abdominal pain, palpable supra-pubic mass and primary amenorrhea. Ultrasound show; well-defined echogenic fluid collection posterior to the urinary bladder, fluid with internal echoes in the uterine cavity the final diagnosis hematometrocolpos due to imperforate hymen.
\end{abstract}

Keywords: Imperforate Hymen, Haematometra, Vagina, Ultrasound

\section{Introduction}

Imperforate hymen is at the extreme of a spectrum of variations in hymenal configuration. Variations in the embryologic development of the hymen are common and result in fenestrations, septa, bands, microperforations, anterior displacement, and differences in rigidity and/or elasticity of the hymenal tissue. Inspection of the external genitalia and anus are important components of the physical examination of the female neonate and child [1].

Affected newborns may present with acute urinary retention [2]. In adolescent females, the most common symptoms of an imperforate hymen are cyclic pelvic pain and amenorrhea; other symptoms associated with hematocolpos include urinary retention, constipation, back pain, nausea, and diarrhea [3].

Diagnosis, an imperforate hymen is most often diagnosed in adolescent girls after the age of menarche with otherwise normal development [4]. In adolescent girls of menarcheal age, the typical presentation of the condition is amenorrhea and cyclic pelvic pain, indicative of hematocolpos secondary to vaginal obstruction. An imperforate hymen is usually visible on vaginal inspection as a bulging blue membrane [4]. If hematocolpos is present, a mass is often palpable on abdominal or rectal examination. The diagnosis of an imperforate hymen is usually made based purely on the physical exam, although if necessary the diagnosis can be confirmed by transabdominal, transperineal or transrectal ultrasound [3]. An imperforate hymen can also be diagnosed in newborn babies and it is occasionally detected on ultrasound scans of the fetus during pregnancy [4]. In newborns the diagnosis is based on the findings of an abdominal or pelvic mass or a bulging hymen[4] Examination of the normal neonatal vagina usually reveals a track of mucus at the posterior commissure of the labia majora; an absence of mucus may indicate an imperforate hymen or another vaginal obstruction [2]. A similar condition, cribriform hymen, is diagnosed when the hymen contains many minute openings [5].

The differential diagnosis of imperforate hymen is from labial adhesions, vaginal atresia, vaginal agenesis, and transverse vaginal septum. However, the abdominal mass has to be diagnosed differentially from distental urinary bladder, ovarian tumors and neoplasms, mesenteric cysts, anterior 
meningocele, reduplication of sigmoid and sacral tumors [6].

\section{Technique}

A low-frequency curvilinear or phased-array transducer or a linear high-frequency transducer may be used for visualization of the abdominal and pelvic structures. The transducer is initially placed in the transverse plane above the pubic bone. Visualization of the bladder may serve as a landmark for locating other pelvic structures. This common sonographic view will quickly identify abnormal anatomy when a mass is palpated in the lower abdomen. Acquiring images in the transverse and sagittal planes as well as defining all borders of each structure can achieve a thorough interrogation, both qualitative and quantitative. When urinary tract obstruction is suspected, obtaining views of the right upper quadrant and left upper quadrant of the abdomen allows for assessment of the kidneys. The kidneys may demonstrate various degrees of hydronephrosis, where the calyces and the renal pelvis become distended and fluid filled in the pelvic view, the bladder may be observed for urine jets, indicating appropriate drainage of urine from the ureters. Finally, the bladder itself can be assessed for gross distension if there is bladder outlet obstruction [7].

\section{Case Report}

We report a case of 16 year old, female, single of Sudan origin that was seen in the ultrasound department with cyclic, lower abdominal pain, palpable supra-pubic mass and primary amenorrhea. A careful ultrasound assessment show; on transverse a well-defined echogenic fluid collection posterior to the urinary bladder $=$ hematocolpos. On sagittal there is small amount of fluid with internal echoes in the uterine cavity $=$ the final diagnosis hematometrocolpos due to imperforate hymen. (FIG I, 2).

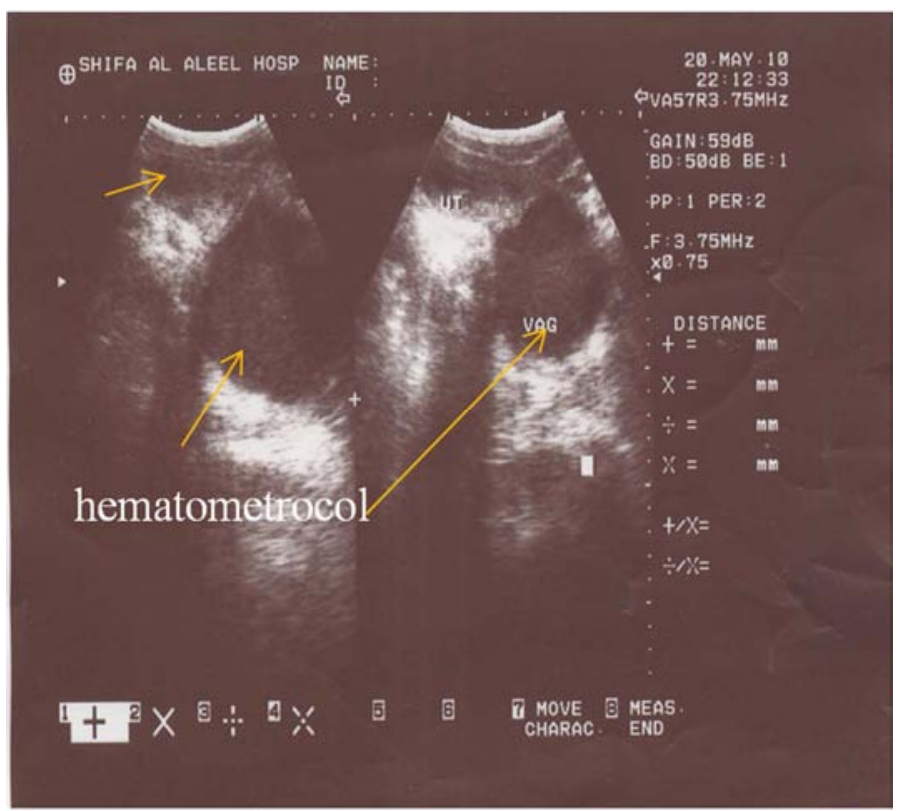

Fig. 1. TAS showing hematometrocolpos, uterus and vagina.

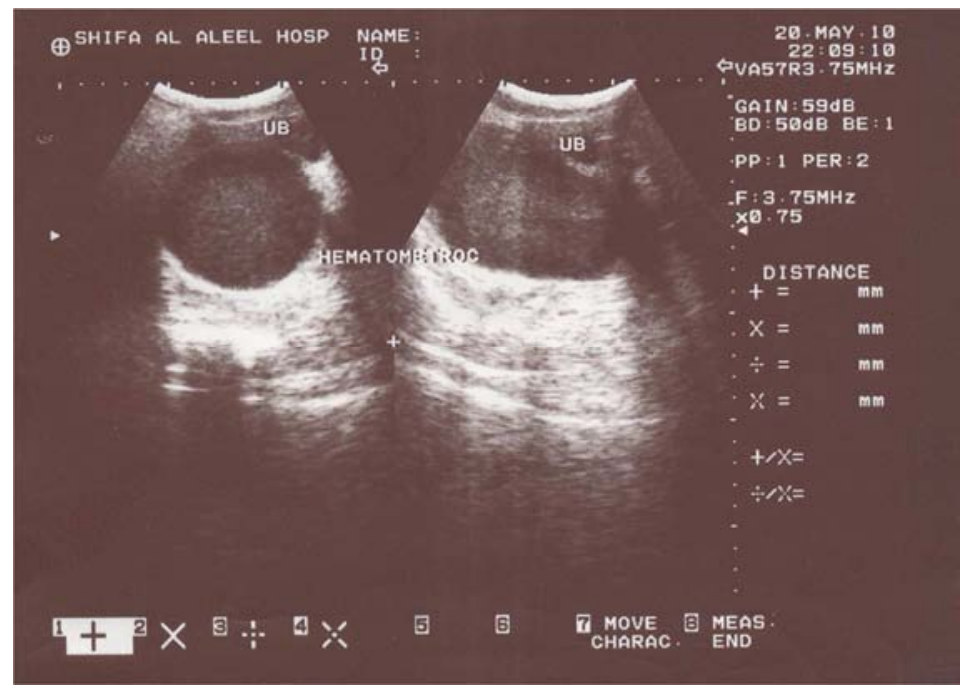

Fig. 2. TAS showing hematometrocolpos. 


\section{Discussion}

In the embryological period, the lateral portion of the hymen originates from a fold of the urogenital sinus. The posterior part originates from the cells of the urogenital sinus, externally, and from Müller's duct, internally. Usually, at the 8th week of gestation, it partially ruptures in the inferior part of Müller's duct, remaining as a fold of mucous membrane around the entrance of the vagina. Failure of these events results in persistence of the septum [8].

Imperforate hymen is rarely diagnosed in the neonatal period, and most cases are presented to the gynecologist between the age of 11 and 15 . Cyclical pelvic pain 2.5 to 4 years after thelarche, primary amenorrhea and a palpable pelvic mass represent the characteristic clinical symptomatology. Urological problems related to external compression of the bladder and ureter is also very common. Urinary hesitancy and dysuria are detected in as many as $58 \%$ of patients with hematocolpos. Although clinical examination is in most cases sufficient for the diagnosis of imperforate hymen, imaging studies are also necessary to confirm the presence of hematocolpos or hematometra [8].

Transabdominal USG is the commonest method used for that purpose2-5. However, in some occasions findings can be misinterpreted as a suspicious pelvic mass and provoke severe anxiety to a young girl and her family. In addition, to further clarify spurious findings of transabdominal USG and rule out possible associated congenital anomalies, other imaging or invasive modalities have been used such as CT scan, MRI scan and video laparoscopy 2-4. All of them are very accurate in diagnosis of hematocolpos and hematometra, however, they are not considered to be cost - effective. Transrectal USG has also been reported as a method of evaluating pelvic anatomy in young girls with no sexual activity. Although, it has been quite accurate in diagnosis of hematocolpos, it is not considered to be an acceptable method for this age group, due to possible psychological implications [8].

The most common symptoms of an imperforate hymen are cyclical abdominal pain and urinary retention, usually presenting between the ages of 13 and 15 years (when menarche occurs) $[9,10]$. There is primary amenorrhea but secondary sex characteristics are well developed. Because the vaginal outflow is obstructed by the non-perforated hymen, menstrual blood accumulates in the vagina (haematocolpos) and the uterus (haematometra). This may lead to mechanical effects on the urethra, bladder, intestines or pelvic blood vessels which can result in urinary retention, obstipation or edema of the legs $[9,11,12]$. Irritation of the sacral plexus or nerve roots can cause lower back pain [13]. Problems with intercourse are rarely mentioned, probably because most of the patients are still sexually inactive [14].

Treatment; before surgical intervention in adolescents, symptoms can be relieved by the combined oral contraceptive pill taken continuously to suppress the menstrual cycle or NSAIDs to relieve pain [15]. Surgical treatment of the imperforate hymen by hymenotomy typically involves making cruciate incisions in the hymen, excising segments of hymen from their bases, and draining the vaginal canal and uterus [16]. For affected girls who wish (or whose parents wish) to have their hymens preserved, surgical techniques to excise of a central flange of the hymen can be used [17]. The timing of surgical hymen repair is controversial: some doctors believe it is best to intervene immediately after the neonatal period, while others believe that surgical repair should be delayed until puberty, when estrogenization is complete [18].

\section{Conclusion}

Girls with acute urinary retention particularly those who have not yet stated menstruation or delayed in their midteens, imperforate hymen should be considered as a possible differential diagnosis.

\section{References}

[1] Braverman PK, Breech L,. American Academy of Pediatrics. Clinical report--gynecologic examination for adolescents in the pediatric office setting. Pediatrics. Sep 2010;126 (3): 58390. [Medline].

[2] Kaiser, Georges L. (2012). Symptoms and Signs in Pediatric Surgery. Springer Science Business Media. p. 556. ISBN 9783642311611.

[3] Lacy, Judith (2008). "Imperforate hymen". The 5-minute Obstetrics and Gynecology Consult. Lippincott Williams \& Wilkins. pp. 116-117. ISBN 9780781769426.

[4] Puri, Prem; Höllwarth, Michael E. (2009). Pediatric Surgery: Diagnosis and Management. Springer Science Business Media. p. 969. ISBN 9783540695608.

[5] Jason W. Fischer, MD and Charisse W. Kwan, MD, Emergency Point-of-Care Ultrasound Diagnosis of Hematocolpometra and Imperforate Hymen in the Pediatric Emergency Department, From the Division of Pediatric Emergency Medicine, Ultrasound Program, The Hospital for Sick Children; and University of Toronto, Toronto, Ontario, Canada, Pediatric Emergency Care \& Volume 30, Number 2, February 2014.

[6] Emmanouil Karteris, Helen Foster, Maria Karamouti and Anastasia Goumenou, Congenital Imperforate Hymen with Hydrocolpos and Hydronephrosis associated with Severe Hydramnios and Increase of Maternal Ovarian Steroidogenic Enzymes, J Pediatr Adolesc Gynecol (2009), North American Society for Pediatric and Adolescent Gynecology Published by Elsevier Inc.

[7] Sharma, R. K. (2007). Concise Textbook Of Forensic Medicine \& Toxicology. Elsevier. p. 117. ISBN 9788131211458 .

[8] Zacharakis Dimitrios, Domali Ekaterini, Antsaklis Panos, Daskalakis George, Imperforate hymen with hematocolpometra: A new diagnostic approach using 2D and 3D translabial ultrasonography, An Obstetrics and Gynecology International Journal, VOLUME 13, ISSUE 2, APRIL - MAY 2014. 
[9] Hall DJ. An unusual case of urinary retention due to imperforate hymen. J Accid Emerg Med 1999; 16: 232-3 [PMC free article] [PubMed].

[10] Letts M, Haasbeek J. Hematocolpos as a cause of back pain in premenarchal adolescents. J Pediatr Orthop 1990; 10: 731. [PubMed].

[11] Dickson CA, Saad S, Tesar JD. Imperforate hymen with hematocolpos. Ann Emerg Med 1985; 14: 467-9 [PubMed].

[12] Yu TJ, Lin MC. Acute urinary retention in two patients with imperforate hymen. Scand J Urol Nephrol 1993; 27: 543-4 [PubMed].

[13] Liang CC, Chang SD, Soong YK. Long-term follow-up of women who underwent surgical correction for imperforate hymen. Arch Gynecol Obstet 2003; 269: 5-8 [PubMed].
[14] Adams Hillard, Paula J. (June 12, 2013). "Imperforate Hymen Treatment \& Management: Medical Therapy". eMedicine. Retrieved May 9, 2014.

[15] Wilkinson, Edward J. (2012). Wilkinson and Stone Atlas of Vulvar Disease (3rd ed.). Lippincott Williams \& Wilkins. pp. 187-188. ISBN 9781451132182.

[16] Chelli D; Kehila M; Sfar E; Zouaoui B; Chelli H; Chanoufi B (2008). "Imperforate hymen: Can it be treated without damaging the hymenal structure?". Santé 18 (2): 83-87. doi: 10.1684/san.2008.0108. PMID 19188131.

[17] Gibbs, Ronald S. (2008). Danforth's Obstetrics and Gynecology. Lippincott Williams \& Wilkins. p. 557. ISBN 9780781769372. 